Article

\title{
A Note on Hadamard Fractional Differential Equations with Varying Coefficients and Their Applications in Probability
}

\author{
Roberto Garra ${ }^{1, *}$, Enzo Orsingher ${ }^{1}$ and Federico Polito ${ }^{2}$ \\ 1 Dipartimento di Scienze Statistiche, "Sapienza” Università di Roma, P. le A. Moro 5, 00185 Roma, Italy; \\ enzo.orsingher@uniroma1.it \\ 2 Dipartimento di Matematica “G. Peano", Università degli Studi di Torino, Via Carlo Alberto 10, \\ 10123 Torino, Italy; f.polito@gmail.com \\ * Correspondence: roberto.garra@sbai.uniroma1.it
}

Received: 18 November 2017; Accepted: 26 December 2017; Published: 1 January 2018

\begin{abstract}
In this paper, we show several connections between special functions arising from generalized Conway-Maxwell-Poisson (COM-Poisson) type statistical distributions and integro-differential equations with varying coefficients involving Hadamard-type operators. New analytical results are obtained, showing the particular role of Hadamard-type derivatives in connection with a recently introduced generalization of the Le Roy function. We are also able to prove a general connection between fractional hyper-Bessel-type equations involving Hadamard operators and Le Roy functions.
\end{abstract}

Keywords: Hadamard fractional derivatives; COM-Poisson distributions; Modified MittagLeffler functions

MSC: 33E12; 34A08; 60G55

\section{Introduction}

The analysis of fractional differential equations involving Hadamard fractional derivatives has increased interest in mathematical analysis, as proved, for example, by the publication of the recent monograph [1]. On the other hand, few results regarding the applications of Hadamard fractional differential equations in mathematical physics (see, for example, [2] and probability (see [3]) exist. In [4], some analytical results regarding Hadamard fractional equations with time-varying coefficients have been pointed out. In particular, the following $\alpha$-Mittag-Leffler function was introduced:

$$
E_{\alpha ; v, \gamma}(z)=\sum_{k=0}^{\infty} \frac{z^{k}}{[\Gamma(v k+\gamma)]^{\alpha}}, \quad z \in \mathbb{C}, \alpha, v, \gamma \in \mathbb{C}
$$

The $\alpha$-Mittag-Leffler function is an entire function of the complex variable $z$ if the parameters are such that $\Re(v)>0, \gamma \in \mathbb{R}$ and $\alpha \in \mathbb{R}^{+}$(see [5]). This function was independently introduced and studied by Gerhold in [6]. In [4], we called this special function $\alpha$-Mittag-Leffler function, since it includes for $\alpha=1$ the well-known two-parameters Mittag-Leffler function

$$
E_{v, \gamma}(z)=\sum_{k=0}^{\infty} \frac{z^{k}}{\Gamma(v k+\gamma)}, \quad z \in \mathbb{C}, v \in \mathbb{C}, \Re(v)>0, \gamma \in \mathbb{R}
$$


widely used in the theory of fractional differential equations (see the recent monograph [7] and the references therein). Moreover, the $\alpha$-Mittag-Leffler function is a generalization of the so-called Le Roy function [8]

$$
R_{\rho}(z)=\sum_{k=0}^{\infty} \frac{z^{k}}{[(k+1) !]^{\alpha}}, \quad z \in \mathbb{C}, \alpha>0 .
$$

In the more recent paper [5], the authors studied the asymptotic behavior and numerical simulation of this new class of special functions.

We observe that the Le Roy functions are used in probability in the context of the studies of COM-Poisson distributions [9], which are special classes of weighted Poisson distributions (see for example [10]). We show in Section 3, as a first probabilistic application, that the $\alpha$-Mittag-Leffler functions can also be used in the construction of a new generalization of the COM-Poisson distribution that can be interesting for statistical applications and in physics in the context of generalized coherent states [11].

The aim of this paper is to study some particular classes of Hadamard fractional integral equations and differential equations whose solutions can be written in terms of the $\alpha$-Mittag-Leffler function (1) and are somehow related to a fractional-type generalization of the COM-Poisson distribution.

A second application in probability is contained in Section 4. We observe that Imoto [12] has recently introduced the following generalization of the COM-Poisson distribution,

$$
P\{N(t)=k\}=\frac{(\Gamma(v+k))^{r} t^{k}}{k ! C(r, v, t)}, \quad k \in \mathbb{N},
$$

involving the normalizing function

$$
C(r, v, t)=\sum_{k=0}^{\infty} \frac{(\Gamma(v+k))^{r} t^{k}}{k !},
$$

with $r<1 / 2, t>0$ and $1>v>0$ or $r=1, v=1$ and $|t|<1$. The special function (5) is somehow related to the generalization of the Le Roy function, while the distribution (4) includes the COM-Poisson for $v=1$ and $r=1-n, n \in \mathbb{R}^{+}$. In Section 4, we show that this function is related to integral equations with a time-varying coefficient involving Hadamard integrals.

In Section 5, we present other results concerning the relation between Le Roy-type functions and Hadamard fractional differential equations. We introduce a wide class of integro-differential equations extending the hyper-Bessel equations. This is a new interesting approach in the context of the mathematical studies of fractional Bessel equations (we refer for example to the recent paper [13] and the references therein).

In conclusion, the main aim of this paper is to establish a connection between some generalizations of the COM-Poisson distributions and integro-differential equations with time-varying coefficients involving Hadamard integrals or derivatives. As a by-product we suggest a possible application of the $\alpha$-Mittag-Leffler function to build a generalized COM-Poisson distribution that in future should be investigated in more detail.

\section{Preliminaries About Fractional Hadamard Derivatives and Integrals}

Starting from the seminal paper by Hadamard [14], many papers have been devoted to the analysis of fractional operators with logarithmic kernels (we refer in particular to [15]). In this section, we briefly recall the definitions and main properties of Hadamard fractional integrals and derivatives and their Caputo-like regularizations recently introduced in the literature. 
Definition 1. Let $t \in \mathbb{R}^{+}$and $\Re(\alpha)>0$. The Hadamard fractional integral of order $\alpha$, applied to the function $f \in L^{p}[a, b], 1 \leq p<+\infty, 0<a<b<\infty$, for $t \in[a, b]$, is defined as

$$
\mathcal{J}^{\alpha} f(t)=\frac{1}{\Gamma(\alpha)} \int_{a}^{t}\left(\ln \frac{t}{\tau}\right)^{\alpha-1} f(\tau) \frac{\mathrm{d} \tau}{\tau}
$$

Before constructing the corresponding differential operator we must define the following space of functions.

Definition 2. Let $[a, b]$ be a finite interval such that $-\infty<a<b<\infty$ and let $A C[a, b]$ be the space of absolutely continuous functions on $[a, b]$. Let us denote $\delta=t \frac{\mathrm{d}}{\mathrm{d} t}$ and define the space

$$
A C_{\delta}^{n}[a, b]=\left\{f: t \in[a, b] \rightarrow \mathbb{R} \text { such that }\left(\delta^{n-1} f\right) \in A C[a, b]\right\}
$$

Clearly $A C_{\delta}^{1}[a, b] \equiv A C[a, b]$ for $n=1$.

Definition 3. Let $\delta=t \frac{\mathrm{d}}{\mathrm{d} t}, \Re(\alpha)>0$ and $n=[\alpha]+1$, where $[\alpha]$ is the integer part of $\alpha$. The Hadamard fractional derivative of order $\alpha$ applied to the function $f \in A C_{\delta}^{n}[a, b], 0 \leq a<b<\infty$, is defined as

$$
\mathcal{D}^{\alpha} f(t)=\frac{1}{\Gamma(n-\alpha)}\left(t \frac{\mathrm{d}}{\mathrm{d} t}\right)^{n} \int_{a}^{t}\left(\ln \frac{t}{\tau}\right)^{n-\alpha-1} f(\tau) \frac{d \tau}{\tau}=\delta^{n}\left(\mathcal{J}^{n-\alpha} f\right)(t)
$$

It has been proved (see e.g., Theorem 4.8 in [16]) that in the $L^{p}[a, b]$ space, $p \in[1, \infty)$, $0 \leq a<b<\infty$, the Hadamard fractional derivative is the left-inverse operator to the Hadamard fractional integral, i.e.,

$$
\mathcal{D}^{\alpha} \mathcal{J}^{\alpha} f(t)=f(t), \quad \forall t \in[a, b] .
$$

Analogously to the Caputo fractional calculus, the regularized Caputo-type Hadamard fractional derivative is defined in terms of the Hadamard fractional integral in the following way (see, for example, [17])

$$
\left(t \frac{\mathrm{d}}{\mathrm{d} t}\right)^{\alpha} f(t)=\frac{1}{\Gamma(n-\alpha)} \int_{a}^{t}\left(\ln \frac{t}{\tau}\right)^{n-\alpha-1}\left(\tau \frac{\mathrm{d}}{\mathrm{d} \tau}\right)^{n} f(\tau) \frac{d \tau}{\tau}=\mathcal{J}^{n-\alpha}\left(\delta^{n} f\right)(t)
$$

where $t \in[a, b], 0 \leq a<b<\infty$ and $n-1<\alpha \leq n$, with $n \in \mathbb{N}$. In this paper, we will use the symbol $\left(t \frac{\mathrm{d}}{\mathrm{d} t}\right)^{\alpha}$ for the Caputo-type derivative in order to distinguish it from the Riemann-Liouville type definition (8) and also to underline the fact that essentially it coincides with the fractional power of the operator $\delta=t \frac{\mathrm{d}}{\mathrm{d} t}$. Moreover, by definition, when $\alpha=n,\left(t \frac{\mathrm{d}}{\mathrm{d} t}\right)^{\alpha} \equiv \delta^{n}$. The relationship between the Hadamard derivative (8) and the regularized Caputo-type derivative is given by ([17], Equation (12))

$$
\left(t \frac{\mathrm{d}}{\mathrm{d} t}\right)^{\alpha} f(t)=\mathcal{D}^{\alpha}\left[f(t)-\sum_{k=0}^{n-1} \frac{\delta^{k} f\left(t_{0}\right)}{k !} \ln ^{k}\left(\frac{t}{t_{0}}\right)\right], \quad \alpha \in(n-1, n], n=[\alpha]+1 .
$$

In the sequel we will use the following useful equalities (that can be checked by simple calculations),

$$
\begin{gathered}
\left(t \frac{\mathrm{d}}{\mathrm{d} t}\right)^{\alpha} t^{\beta}=\beta^{\alpha} t^{\beta} \\
\mathcal{J}^{\alpha} t^{\beta}=\beta^{-\alpha} t^{\beta}
\end{gathered}
$$


for $\beta \in(-1, \infty) \backslash\{0\}$ and $\alpha>0$. It is immediate to see that

$$
\left(t \frac{\mathrm{d}}{\mathrm{d} t}\right)^{\alpha} \text { const }=0
$$

Finally, we observe that formally the relationship between Hadamard-type derivatives and Riemann-Liouville derivatives is given by the change of variable $t \rightarrow \ln (t)$, leading to the logarithmic kernel. According to this change of variable, the Hadamard derivative represents the counterpart of the fractional power of the operator $\frac{\mathrm{d}}{\mathrm{d} t}$, i.e., the fractional power of the operator $\delta=t \frac{\mathrm{d}}{\mathrm{d} t}$.

\section{Generalized COM-Poisson Processes Involving the $\alpha$-Mittag-Leffler Function and the Related Hadamard Equations}

Here we show a possible application of the $\alpha$-Mittag-Leffler function (1) in the context of fractional-type Poisson statistics and we discuss the relation with Hadamard equations.

We first recall that weighted Poisson distributions have been widely studied in statistics in order to consider over or under-dispersion with respect to the homogeneous Poisson process. The probability mass function of a weighted Poisson process $N^{w}(t), t>0$, is given by (see [10])

$$
P\left\{N^{w}(t)=n\right\}=\frac{w(n) p(n, t)}{\mathbb{E}[w(N)]}, \quad n \geq 0,
$$

where $N$ is a Poisson distributed random variable

$$
p(n, t)=\frac{t^{n}}{n !} e^{-t}, \quad n \geq 0, t>0
$$

$w(\cdot)$ is a non-negative weight function with non-zero, finite expectation, i.e.,

$$
0<\mathbb{E}[w(N)]=\sum_{k=0}^{\infty} w(k) p(k, t)<+\infty .
$$

The so-called COM-Poisson distribution introduced by Conway and Maxwell [9] in a queueing model belongs to this wide class of distributions. A random variable $N_{v}(t)$ is said to have a COM-Poisson distribution if

$$
P\left\{N_{v}(t)=n\right\}=\frac{1}{f(t)} \frac{t^{n}}{n !^{\nu}}, \quad n \geq 0, t>0,
$$

where

$$
f(t)=\sum_{k=0}^{\infty} \frac{t^{k}}{k !^{v}}, \quad v>0,
$$

is the Le-Roy function.

This distribution can be viewed as a particular weighted Poisson distribution with $w(n)=1 /(n !)^{v-1}$. The Conway-Maxwell-Poisson distribution is widely used in statistics in order to take into account over-dispersion (for $0<v<1$ ) or under-dispersion (for $v>1$ ) in data analysis. Moreover it represents a useful generalization of the Poisson distribution (that is obtained for $v=1$ ) for many applications (see for example [18] and the references therein). Observe that when $t \in(0,1)$ and $v \rightarrow 0$ it reduces to the geometric distribution, while for $v \rightarrow+\infty$ it converges to the Bernoulli distribution.

Another class of interesting weighted Poisson distributions are the fractional Poissontype distributions

$$
P\left\{N_{\alpha}(t)=k\right\}=\frac{1}{E_{\alpha, 1}(t)} \frac{t^{k}}{\Gamma(\alpha k+1)}, \quad \alpha \in(0,1),
$$


where

$$
E_{\alpha, 1}(t)=\sum_{k=0}^{\infty} \frac{t^{k}}{\Gamma(\alpha k+1)}
$$

is the Mittag-Leffler function (see the recent monograph [7] for more details). In this case, the weight function is given by $w(k)=k ! / \Gamma(\alpha k+1)$. There is a recent wide literature about fractional generalizations of Poisson processes (see e.g., [19-27]) and we should explain in which sense Equation (19) can be regarded as a fractional-type Poisson distribution. The relationship of this kind of distributions with fractional calculus, first considered by Beghin and Orsingher in [28], is given by the fact that the probability generating function

$$
G(u, t)=\sum_{k=0}^{\infty} u^{k} P\left\{N_{\alpha}(t)=k\right\}=\frac{E_{\alpha, 1}(u t)}{E_{\alpha, 1}(t)}, \quad \alpha \in(0,1],
$$

satisfies the fractional differential equation

$$
\frac{d^{\alpha} G\left(u^{\alpha}, t\right)}{d u^{\alpha}}=t G\left(u^{\alpha}, t\right), \quad \alpha \in(0,1],|u| \leq 1,
$$

where $d^{\alpha} / d u^{\alpha}$ denotes the Caputo fractional derivative w.r.t. the variable $u$ (see [16] for the definition). Essentially, the connection between the distribution (19) and fractional calculus is therefore given by the normalizing function, since the Mittag-Leffler plays the role of the exponential function in the theory of fractional differential equations. In this context, we discuss a new generalization of the COM-Poisson distribution by using the generalized $\alpha$-Mittag-Leffler function Equation (1), independently introduced by Gerhold in [6] and by Garra and Polito in [4]. This new fractional distribution includes a wide class of special distributions already studied in the recent literature: the fractional Poisson distribution Equation (19) considered by Beghin and Orsingher [28], the classical COM-Poisson distribution and so on.

In this paper, we introduce the following generalization, namely the fractional COMPoisson distribution

$$
P\left\{N_{v ; \alpha, \gamma}(t)=n\right\}=\frac{(\lambda t)^{n}}{(\Gamma(\alpha n+\gamma))^{v}} \frac{1}{E_{v ; \alpha, \gamma}(\lambda t)^{\prime}}
$$

where

$$
E_{v ; \alpha, \gamma}(t)=\sum_{k=0}^{\infty} \frac{t^{k}}{(\Gamma(\alpha k+\gamma))^{v}}, \quad \alpha>0, v>0, \gamma \in \mathbb{R}
$$

is the $\alpha$-Mittag-Leffler function, considered by Gerhold [6], Garra and Polito [4]. The distribution Equation (23) is a weighted Poisson distribution with weights $w(k)=k ! /(\Gamma(\alpha k+\gamma))^{\nu}$. Obviously the probability generating function is here given by a ratio of $\alpha$-Mittag-Leffler functions, i.e.,

$$
G(u, t)=\sum_{n=0}^{\infty} u^{n} P\left\{N_{v ; \alpha, \gamma}(t)=n\right\}=\frac{E_{v ; \alpha, \gamma}(\lambda u t)}{E_{v ; \alpha, \gamma}(\lambda t)}, \quad|u| \leq 1 .
$$

With the next theorem we explain the reason why we consider the distribution Equation (23), a kind of fractional generalization of the COM-Poisson classical distribution related to Hadamard fractional equations with varying coefficients.

Theorem 1. For $v \in(0,1)$, the function

$$
g(u, t)=u^{v-1} \frac{E_{v ; 1, v}(\lambda u t)}{E_{v ; 1, v}(\lambda t)}=u^{v-1} G(u, t),
$$


satisfies the equation

$$
\left(u \frac{\mathrm{d}}{\mathrm{d} u}\right)^{v} g(u, t)=\lambda t u g(u, t)+\frac{u^{v-1}}{\Gamma^{v}(v-1)}
$$

where $\left(u \frac{\mathrm{d}}{\mathrm{d} u}\right)^{v}$ is the Caputo-Hadamard fractional derivative of order $v$.

Proof. By direct calculations we obtain that

$$
\begin{aligned}
\left(u \frac{\mathrm{d}}{\mathrm{d} u}\right)^{v} u^{v-1} \frac{E_{v ; 1, v}(\lambda u t)}{E_{v ; 1, v}(\lambda t)} & =\frac{1}{E_{v ; 1, v}(\lambda t)} \sum_{k=0}^{\infty} \frac{\lambda^{k} u^{k+v-1} t^{k}}{(\Gamma(k+v-1))^{v}} \\
& =\frac{1}{E_{v ; 1, v}(\lambda t)} \sum_{k=-1}^{\infty} \frac{\lambda^{k+1} u^{k+v} t^{k+1}}{(\Gamma(k+v))^{v}}=\lambda t u g(u, t)+\frac{u^{v-1}}{(\Gamma(v-1))^{v}}
\end{aligned}
$$

where we changed the order of summation with fractional differentiation since the $v$-Mittag-Leffler function is, for $v \in(0,1)$, an entire function.

A more detailed analysis about the statistical properties and possible applications of this new class of distributions is not the object of this paper and will be considered in a future work.

We finally observe that the fractional COM-Poisson distribution (23) includes as a special case, for $v=1$ the fractional Poisson distribution (19) previously introduced by Beghin and Orsingher in [28] and recently treated by Chakraborty and Ong [29], Herrmann [21] and Porwall and Dixit [30]. Asymptotic results for the multivariate version of this distribution have been recently analyzed by Beghin and Macci in [19].

\section{Hadamard Fractional Equations Related to the GCOM-Poisson Distribution}

In a recent paper, Imoto [12] studied a different generalization of the Conway-Maxwell-Poisson distribution (in the following GCOM Poisson distribution), depending on two real parameters $r$ and $v$. According to [12], a random variable $X(t)$ has a GCOM Poisson distribution if

$$
P\{X(t)=n\}=\frac{(\Gamma(v+n))^{r} t^{n}}{n ! C(r, v, t)}, \quad t \geq 0, n=0,1, \ldots,
$$

where

$$
C(r, v, t)=\sum_{k=0}^{\infty} \frac{(\Gamma(\nu+k))^{r} t^{k}}{k !},
$$

is the normalizing function which converges for $v>0, t>0$ and $r<1 / 2$ (by applying the ratio criterion) or $r=1, v>0$ and $|t|<1$. In the context of weighted Poisson distributions, it corresponds to the choice $w(k)=\Gamma^{r}(v+k)$.

This distribution includes as particular cases the COM-Poisson distribution for $v=1$ and $r=1-n$, the geometric for $r=v=1,|t|<1$ and the homogeneous Poisson distribution for $r=0$. The new parameter introduced in the distribution plays the role of controlling the length of tails within the framework of queueing processes. Indeed it was proved that, it displays over-dispersion for $r \in(0,1)$ and under-dispersion for $r<0$. Moreover, from the ratios of successive probabilities, when $v>1$ and $r \in(0,1)$ it emerges to be heavy tailed, while for $r<0$ light-tailed.

We also underline that for $r=1-n$, with $n>0$, the function (29) becomes a sort of generalization of the Wright function which can be written as

$$
C(1-n, v, t)=\sum_{k=0}^{\infty} \frac{t^{k}}{k ! \Gamma^{n}(v+k)}
$$


Recall that, in the general form, the Wright function is defined as follows (we refer to [31] for a good survey):

$$
W_{\alpha, \beta}(t)=\sum_{k=0}^{\infty} \frac{t^{k}}{k ! \Gamma(\alpha k+\beta)}, \quad \alpha>-1, \beta \in \mathbb{C} .
$$

We show now the relation between the normalizing function Equation (29) and an Hadamard fractional differential equation with time-varying coefficients. Therefore, we can consider in some sense also the GCOM Poisson distribution as a fractional-type modification of the homogeneous distribution, in the sense that its probability generating function satisfies a fractional differential equation.

Proposition 1. The function

$$
C(r, v, t)=\sum_{k=0}^{\infty} \frac{\Gamma^{r}(v+k)(\lambda t)^{k}}{k !}
$$

satisfies the integro-differential equation

$$
\mathcal{J}^{r}\left(t^{\nu} \frac{\mathrm{d} f}{\mathrm{~d} t}\right)=\lambda t^{\nu} f, \quad t>0, v \in(0,1], r \in(0,1 / 2)
$$

involving an Hadamard fractional integral $\mathcal{J}^{r}$ of order $r$.

Proof.

$$
\begin{aligned}
\mathcal{J}^{r}\left(t^{v} \frac{\mathrm{d} C}{\mathrm{~d} t}\right) & =\mathcal{J}^{r} \sum_{k=1}^{\infty} \frac{\Gamma^{r}(v+k) \lambda^{k} t^{k+v-1}}{(k-1) !}=\sum_{k=1}^{\infty} \frac{\Gamma^{r}(v+k)(k+v-1)^{-r} \lambda^{k} t^{k+v-1}}{(k-1) !} \\
& =\sum_{k=1}^{\infty} \frac{\Gamma^{r}(v+k-1) \lambda^{k} t^{k+v-1}}{(k-1) !}=\lambda t^{v} f
\end{aligned}
$$

where we used Equation (13).

Corollary 1. The probability generating function $\mathcal{G}(u, t)$ of the GCOM Poisson distribution Equation (28)

$$
\mathcal{G}(u, t)=\sum_{k=0}^{\infty} u^{k} P\{X(t)=k\}=\frac{C(r, v, u t)}{C(r, v, t)}, \quad|u| \leq 1,
$$

satisfies the integro-differential equation

$$
\mathcal{J}^{r}\left(u^{\nu} \frac{d f}{d u}\right)=u^{v} f, \quad|u| \leq 1
$$

\section{Further Connections between Modified Mittag-Leffler Functions and Hadamard Fractional Equations}

The analysis of fractional differential equations with non-constant coefficients is an interesting and non-trivial topic. In particular, the analysis of equations involving fractional-type Bessel operators (i.e., the fractional counterpart of singular linear differential operators of arbitrary order) has attracted the interest of many researchers (see e.g., [13,32] and references therein). In [4], some results about the connection between Le-Roy functions and equations with space-varying coefficients involving Hadamard derivatives and Laguerre derivatives have been obtained. Here we go further in the direction started in [4], showing other interesting applications of Hadamard fractional equations in the theory of hyper-Bessel functions. With the next theorem, we find the equation interpolating classical Bessel equations of arbitrary order possessing exact solution in terms of Le Roy functions. We remark, indeed, that Le Roy functions include as special cases hyper-Bessel functions. 
Theorem 2. The Le Roy function $E_{\alpha ; 1,1}\left(t^{\alpha} / \alpha\right)$ satisfies the equation

$$
\frac{1}{t^{\alpha}}\left(t \frac{\mathrm{d}}{\mathrm{d} t}\right)^{\alpha} f(x)=\alpha^{\alpha-1} f(t)
$$

Proof. Recall that

$$
\left(t \frac{\mathrm{d}}{\mathrm{d} t}\right)^{\alpha} t^{\beta}=\beta^{\alpha} t^{\beta}
$$

Therefore

$$
\left(t \frac{\mathrm{d}}{\mathrm{d} t}\right)^{\alpha} E_{\alpha ; 1,1}\left(\frac{t^{\alpha}}{\alpha}\right)=\sum_{k=0}^{\infty} \frac{(\alpha k)^{\alpha} t^{\alpha k}}{\alpha^{k} k !^{\alpha}}=\alpha^{\alpha-1} t^{\alpha} E_{\alpha ; 1,1}\left(\frac{t^{\alpha}}{\alpha}\right) .
$$

Remark 1. For $\alpha=2$, we have that the function $E_{2 ; 1,1}\left(t^{2} / 2\right)$ is a solution of

$$
\left(t \frac{\mathrm{d}}{\mathrm{d} t}\right)^{2} f(t)=2 t^{2} f(t)
$$

For $\alpha=3$, the function $E_{3 ; 1,1}\left(t^{3} / 3\right)$ satisfies

$$
\left(t \frac{\mathrm{d}}{\mathrm{d} t}\right)^{3} f(t)=9 t^{3} f(t)
$$

and so forth for any integer value of $\alpha$. From this point of view, the solution of Hadamard Equation (37), for non integer values of $\alpha$, leads to an interpolation between successive hyper-Bessel functions.

Let us consider the operator

$$
L_{H}=\frac{1}{t^{\alpha n}} \underbrace{\left(t \frac{\mathrm{d}}{\mathrm{d} t}\right)^{\alpha}\left(t \frac{\mathrm{d}}{\mathrm{d} t}\right)^{\alpha} \ldots\left(t \frac{\mathrm{d}}{\mathrm{d} t}\right)^{\alpha}}_{n \text { times }}, \quad \alpha>0,
$$

where $H$ stands for an Hyper-Bessel type operator involving Caputo-type Hadamard derivatives. We have the following general Theorem

Theorem 3. A solution of the equation

$$
L_{H} f(t)=\alpha^{n \alpha-n} n^{n \alpha} f(t),
$$

is given by

$$
f(t)=E_{n \alpha ; 1,1}\left(t^{\alpha n} / \alpha^{n}\right)
$$

To conclude this section, we restate Theorem 3.3 of [4], in view of the comments of Turmetov [33] in the case in which Caputo-type Hadamard derivatives appear in the governing equations.

Theorem 4. The function $E_{\beta ; 1,1}(t)$, with $\beta \in[1, \infty), t \geq 0, \lambda \in \mathbb{R}$ is an eigenfunction of the operator

$$
\underbrace{\frac{\mathrm{d}}{\mathrm{d} t} t \frac{\mathrm{d}}{\mathrm{d} t} \ldots \frac{\mathrm{d}}{\mathrm{d} t} t \frac{\mathrm{d}}{\mathrm{d} t}}_{r \text { derivatives }}\left(t \frac{\mathrm{d}}{\mathrm{d} t}\right)^{\beta-r}, \quad r=1, \ldots, n-1,
$$


where $n=[\beta]$ is the integer part of $\beta$ and $\left(t \frac{\mathrm{d}}{\mathrm{d} t}\right)^{\beta-r}$ denotes the Caputo-type regularized Hadamard derivative of order $\beta-r$.

The difference w.r.t. the previous version is simply given by the fact that, by using the regularized Caputo-type Hadamard derivative $\left(t \frac{\mathrm{d}}{\mathrm{d} t}\right)^{\alpha} t^{0}=0$, that is necessary for the correctness of the result.

Acknowledgments: The work of R.G. has been carried out in the framework of the activities of GNFM. F.P. has been supported by the projects Memory in Evolving Graphs (Compagnia di San Paolo/Università di Torino), Sviluppo e analisi di processi Markoviani e non Markoviani con applicazioni (Università di Torino), and by INDAM-GNAMPA.

Author Contributions: The authors contributed equally to this work.

Conflicts of Interest: The authors declare no conflict of interest.

\section{References}

1. Ahmad, B.; Alsaedi, A.; Ntouyas, S.K.; Tariboon, J. Hadamard-Type Fractional Differential Equations, Inclusions and Inequalities; Springer: Basel, Switzerland, 2017.

2. Garra, R.; Giusti, A.; Mainardi, F.; Pagnini, G. Fractional relaxation with time-varying coefficient. Fract. Calc. Appl. Anal. 2014, 17, 424-439.

3. Saxena, R.K.; Garra, R.; Orsingher, E. Analytical solution of space-time fractional telegraph-type equations involving Hilfer and Hadamard derivatives. Integral Transform. Spec. Funct. 2016, 27, 30-42.

4. Garra, R.; Polito, F. On Some Operators Involving Hadamard Derivatives. Integral Transform Spec. Funct. 2013, 24, 773-782.

5. Garrappa, R.; Mainardi, F.; Rogosin, S.V. On a generalized three-parameter Wright function of Le Roy-type. Fract. Calc. Appl. Anal. 2017, 20, 1196-1215.

6. Gerhold, S. Asymptotics for a variant of the Mittag-Leffler function. Integral Transform Spec. Funct. 2012, 23, 397-403.

7. Gorenflo, R.; Kilbas, A.A.; Mainardi, F.; Rogosin, S.V. Mittag-Leffler Functions. Related Topics and Applications; Springer Monographs in Mathematics; Springer: Berlin, Germany, 2014.

8. Le Roy, E. Valeurs asymptotiques de certaines séries procédant suivant les puissances entéres et positives d'une variable réelle. Darboux Bull. 1899, 24, 245-268. (In French).

9. Conway, R.W.; Maxwell, W.I. A queueing model with state dependent service rate. J. Ind. Eng. 1961, 12, 132-136.

10. Balakrishnan, N.; Kozubowski, T.J. A class of weighted Poisson processes. Stat. Probab. Lett. 2008, 78, 2346-2352.

11. Sixdeniers, J.M.; Penson, K.A.; Solomon, A.I. Mittag-Leffler coherent states. J. Phys. A 1999, 32, 7543-7563.

12. Imoto, T. A generalized Conway-Maxwell-Poisson distribution which includes the negative binomial distribution. Appl. Math. Comput. 2014, 247, 824-834.

13. Shishkina, E.L.; Sitnik, S.M. On fractional powers of Bessel operators. J. Inequal. Spec. Funct. 2017, 8, 49-67.

14. Hadamard, J. Essai sur l'etude des fonctions donnees par leur developpment de Taylor. J. Math. Pures Appl. 1892, 8, 101-186.

15. Kilbas, A.A. Hadamard-type fractional calculus. J. Korean Math. Soc. 2001, 38, 1191-1204.

16. Kilbas, A.A.; Srivastava, H.M.; Trujillo, J.J. Theory and Applications of Fractional Differential Equations; Elsevier: Amsterdam, The Netherlands, 2006; Volume 204.

17. Jarad, F.; Abdeljawad, T.; Baleanu, D. Caputo-type modification of the Hadamard fractional derivative. Adv. Differ. Equ. 2012, 1-8, doi:10.1186/1687-1847-2012-142.

18. Rodado, A.; Bebbington, M.; Noble, A.; Cronin, S.; Jolly, G. On selection of analog volcanoes. Math. Geosci. 2011, 43, 505-519.

19. Beghin, L.; Macci, C. Asymptotic results for a multivariate version of the alternative fractional Poisson process. Stat. Probab. Lett. 2017, 129, 260-268.

20. Beghin, L.; Ricciuti, C. Time-inhomogeneous fractional Poisson processes defined by the multistable subordinator. arXiv 2016, arXiv:1608.02224. 
21. Herrmann, R. Generalization of the fractional Poisson distribution. Fract. Calc. Appl. Anal. 2006, 19, 832-842.

22. Laskin, N. Fractional Poisson process. Commun. Nonlinear Sci. Numer. Simul. 2003, 8, 201-213.

23. Mainardi, F.; Gorenflo, R.; Scalas, E. A fractional generalization of the Poisson processes. Vietnam J. Math. 2013, 32, 53-64.

24. Meerschaert, M.; Nane, E.; Vellaisamy, P. The fractional Poisson process and the inverse stable subordinator. Electron. J. Probab. 2011, 16, 1600-1620.

25. Orsingher, E.; Toaldo, B. Counting processes with Bernstein intertimes and random jumps. J. Appl. Probab. 2015, 52, 1028-1044.

26. Orsingher, E.; Polito, F. The space-fractional Poisson process. Stat. Probab. Lett. 2012, 82, 852-858.

27. Polito, F.; Scalas, E. A Generalization of the Space-Fractional Poisson Process and its Connection to some Lévy Processes. Electron. Commun. Probab. 2016, 21, 1-14.

28. Beghin, L.; Orsingher, E. Fractional Poisson processes and related planar random motions. Electron. J. Probab. 2009, 14, 1790-1826.

29. Chakraborty, S.; Ong, S. H. Mittag-Leffler function distribution-A new generalization of hyper-Poisson distribution. J. Stat. Distrib. Appl. 2017, 4, 8, doi:10.1186/s40488-017-0060-9.

30. Porwal, S.; Dixit, K.K. On Mittag-Leffler type Poisson distribution. Afr. Mat. 2017, 28, 29-34.

31. Gorenflo, R.; Luchko, Y.; Mainardi, F. Analytical properties and applications of the Wright function. Fract. Calc. Appl. Anal. 1999, 2, 383-414.

32. Kiryakova, V.S. Generalized Fractional Calculus and Applications; CRC Press: Boca Raton, FL, USA, 1993.

33. Turmetov, B.K. On certain operator method for solving differential equations. AIP Conf. Proc. 2015, 1676, doi:10.1063/1.4930520.

(C) 2018 by the authors. Licensee MDPI, Basel, Switzerland. This article is an open access article distributed under the terms and conditions of the Creative Commons Attribution (CC BY) license (http:// creativecommons.org/licenses/by/4.0/). 